\title{
Linear Local Order
}

\author{
BY R. COMÈS
}

Laboratoire de Physique des Solides associé au CNRS, Bâtiment 510, Université Paris-Sud, 91405 Orsay, France

(Received 10 June 1974)

\begin{abstract}
If the occurrence of one or two-dimensional local order in alloys or in neutron-irradiated materials has been known for many years, the observation by X-ray and neutron scattering of quasi-one-dimensional order due to displacements or correlated motions of atoms along independent chains is more recent. Examples connected with ferroelectric phase transitions in perovskite crystals [Harada, J., Motegi, H., Honjo, G., Mitsui, S. \& Hoshino, S. (1967). J. Phys. Soc. Japan, 22, 1515; Comès, R., Lambert, M. \& Guinier, A. (1970). Acta Cryst. A26, 244; Hüller, A. (1969). Z. Phys. 220, 145; Yamada, Y. \& Shirane, G. (1969). Phys. Rev. 177, 848; Comès, R. \& Shirane, G. (1972). Phys. Rev. B5, 1886; Currat, R., Comès, R., Dorner, B. \& Wiesendanger, E. (1975). J. Phys. C. To be published], where the origin of such one-dimensional phenomena is still obscure, and the case of the one-dimensional metals $\left(\mathrm{K}_{2} \mathrm{Pt}(\mathrm{CN})_{4} \mathrm{Br}_{0 \cdot 30} \cdot 3 \mathrm{H}_{2} \mathrm{O}\right)$ where the one-dimensional order is a direct consequence of the coupling of the phonons to the one-dimensional electrons at the Fermi surface [Zeller, H. R. (1973). Festkörperprobleme, Edited by H. J. Queisser, Vol. 13, p. 31. New York: Pergamon Press; Krogmann, K. \& Hausen, H. D. (1968). Z. Anorg. Chem. 358, 67; Comès, R., Lambert, M., Launois, H. \& Zeller, H. R. (1973). Phys. Rev. B8, 571; Renker, B., Rietschel, H., Pintschovius, L., Gläser, W., Briiesch, P., Kuse, D. \& Rice, M. J. (1973). Phys. Rev. Lett. 30, 1144; Comès, R., Lambert, M. \& Zeller, H. R. (1973). Phys. Stat. Sol. (b), 58, 587; Renker, B., Pintschovius, L., Gläser, W., Rietschel, H., Comès, R., Liebert, L. \& Drexel, W. (1974). Phys. Rev. Lett. 32, 836; Bertinotti, A., Bertinotti, C. \& Jehanno, G. (1974). C. R. Acad. Sci. Paris, sér. B, 278, 45] will be discussed.
\end{abstract}

J. Appl. Cryst. (1975). 8, 132

\section{On the Problem of Fluctuations near First-Order Phase Transformations in Solids}

\author{
BY H. E. COOK \\ Departments of Metallurgy and Mechanical Engineering, University of Illinois at Urbana-Champaign, \\ Urbana, Illinois 61801, U.S.A.
}

(Received 10 June 1974; accepted 7 September 1974)

\begin{abstract}
The recent experimental results on the phonon structure of b.c.c. $\mathrm{Zr}-\mathrm{Nb}$ alloys are reviewed. The central (elastic) phonon peak is discussed in terms of quasi-static heterophase fluctuations which are postulated to form by thermal excitation to a coherent-metastable state and to decay by boundary collapse. This concept is extended qualitatively to the problem of fluctuations associated with first-order compositional transformations - clustering and ordering. It is suggested that quasi-static fluctuations should also occur near the coherent phase boundary for first-order compositional transformations. These fluctuations can be properly interpreted as small particles of the low-temperature phase. The model is used to discuss qualitatively the disorder to long-period and long-period to ideally ordered phase transitions.
\end{abstract}

\section{Introduction}

The general phenomenological theory of phase transformations given by Landau \& Lifshitz (1958)* enables one to decide on the basis of the change in symmetry if a transformation can be second order. The symmetry change must be such that the structure of the low-temperature phase is stabilized at an arbitrarily small value of the order parameter $\eta$. For the first-order

* Referred to here as the Landau theory (Landau, 1937). reaction, however, the low-temperature phase only becomes stable at a finite value of the order parameter. A consequence is that a metastable state is formed above the transformation temperature. Most of the features usually given to distinguish between the transformations can be related to the existence or absence of the metastable state.

The free energy, $F$, shown separately in Fig. 1 for firstand second-order transformations is based on the Taylor expansion

$$
F=A \eta^{2}+B \eta^{3}+C \eta^{4}+\ldots
$$

